\title{
3D Simulation System for Physical Lab using an Object Oriented Approach
}

\author{
Zeena Abdulateef Salman \\ Computer Science Dept. \\ College of Science \\ Al- Mustansiriyah University \\ Baghdad, IRAQ
}

\author{
Karim Q. Hussein, PhD \\ Computer Science Dept. \\ College of Science \\ Al- Mustansiriyah University \\ Baghdad, Iraq
}

\begin{abstract}
This paper presents a practical approach for 3D Animated Modules of physics experiments for third $3^{\text {rd }}$ secondary class in Iraqi schools. The performance of the 3D simulation system was built and tested by using object oriented software engineering (OOSE) in evaluating it . 3D computer graphics, imaging system, Modeling, Animation using Autodesk Maya 2014 program and Its algorithms and models are used to implement the 3D vision of the experiments in creating 3D images with very high resolution. The proposed system of 3D animated modules allows simulation for physics experiments in easy and repeatable manner. The newly designed approach can be used in any time such as in home .By using this designed model ,the student can easily listen to the lessons of the selected specific subject of physics, uses the mathematical model to test the rules of physics and chooses if he wants to take a simple exam.
\end{abstract}

\section{General Terms}

Ray tracing algorithm for realistic images, pipeline graphics model

\section{Keywords}

3D Simulation(3DS), Virtual Learning Environment(VLE), 3D Movie (3DM), Computer Graphics (CG), Instructional Simulation(IS), MAYA

\section{INTRODUCTION}

The new generation of learners, called Net Generation, are more easy with image-rich rather that a text-only condition ${ }^{[1]}$. Old-style ways of teaching physics science are often unsuccessful in attracting studentlinto the self-control. As the world continues to change, continuing to offer the same kinds of education to students will not help them well ${ }^{[2]}$. According to Halloun ${ }^{[3]}$, the use of increasingly sophisticated software encourages a constructivist methodology to learning. 3D Simulation (3DS) applies (VLE) as an approach via engaging problem-solving activities. Students use computer programs to simulate the practical part of student's class (laboratory) that need for special supplies .3DS contains the specification of a mathematical model that is applied several times with different multiple parameters to reveal relationships and illustrate concepts in voice and 3DM.

\section{PROBLEM STATEMENT}

The paper intends to explain how to invest the modeling S.W technology of the 3D Movies (3DMs ) software and develop a systematic computer model using object oriented software engineering to simulate the selected experiments of Electric Circuits (EC) topics as an interactive physics laboratory . This represents a new advanced ways to capture students' responsiveness and make education a more normal and pleasant experience rather than the less attractive traditional approach can be challenging.

\section{MOTIVATION TOWARDS RESEARCH}

According to Lim (2007) digital technologies will chief to a revolution that will change the way of learning in additional to determine what they learn and with whom. A phenomenon that has and remain to affect the technical and qualified educational sector ${ }^{[4]}$.The intended research paper is to explain the using of multimedia technologies, the simulation tools and OOSE in the design for learning process and to provide a possibility for students to repeat the subjects and solve problems by watching and listening to the lessons of the laboratories, whenever and wherever they want .The advantage of this technique is effective, more practical and cost -efficient. The benefit of the research work can be summarized as:

1. Simulate the physics experiments and physics theories in a 3DM way. The advantage of simulation 3DM maybe more $\mathrm{S} / \mathrm{W}$ and best look.

2. The research fetches theories from fields of physics, such as classic physics, to CG. The applications can be used in systems such as virtual training of movies training and visualization.

3. The using of $3 \mathrm{D}$ computer models in the schoolroom may assist increasing from student's ability to understand the new and complex course's subjects, and offer a chance to improve visuospatial skill ${ }^{[1]}$.

4. According to the authors survey, Little work has been done on this work.

5. Deep learning because IS contain the following five elements:-

a. One or more dynamic models.

b. Interaction resulting in state changed.

c. Non_linear logic.

d. Help structure to argument instruction.

e. The pursuit of educational goal. ${ }^{[5]}$

\section{OBJECTIVE OF RESEARCH}

This research work aims at:-

1. Building a systematic complete model for simulation modules of electrical circuits' laboratories or experiments. 
2. Implementing a 3D simulation system to introduce simulation modules for electrical circuit experiments based on the above model.

3. Applying the above system to introduce real 3D simulation modules for selected experiments in electrical circuits

4. Testing the mentioned 3D simulation modules to evaluate the model.

\section{TOOLS, SCOPE AND LIMITATION}

These could be summarized with the following items:-

\subsection{Software}

Several softwares have been used in implementing the simulation system for 3D animated Modules as stated bellow.
a) Autodesk Maya and MEL script.
b) C sharp.
c) Adobe Premiere Pro CC.
d) Tadween.

\subsection{Theories and Algorithms}

Theories and Algorithms have been used in implementing the instructional simulation system for 3D animated Modules stated bellow.

\section{a) Geometric Theory}

The Geometric Theory has been used in modeling to create 3 D objects ${ }^{[6]}$.

\section{b) Autodesk Maya Polygonal Modeling Theory}

The Polygonal Modeling Theory of Autodesk Maya has been used in modeling ${ }^{[7]}$.

c) Rendering Ray trace in Autodesk Maya 2014

The process of tracing rays after they hit the surface and the objects in the scene ${ }^{[8]}$.

\section{d) Computer Graphics}

CGs have been designed by depending on the graphics process that has divided into: geometric modeling, 3D animation definition, rendering, display and image storage. The rendering depends on software (lighting information and texturing information) and hardware (GPU) ${ }^{[9]}$.

\subsection{The Scope of the Research}

The subject of physical experiments of electric circuits' laboratory .The research work covers the following experiments:-

- $\quad$ Electric Circuit.

- Voltmeter Device.

- Parallel Connections /one bulb.

- Parallel connections / two bulbs.

- Parallel connections / three bulbs.

- Series Connection/ one bulb.

- Series Connection/ two bulbs.

- Series Connection/ three bulbs.

- Electric Resistance and its unit.

- The factor affecting the electrical resistance.

\subsection{The Limitations}

The subjects of physical experiments are depending on ten lessons of electric circuit's experiences in physics for third $3^{\text {rd }}$ secondary schools in Iraq.

\section{RELATED WORK}

A. Jeffrey Richardson, Nicoletta Adamo-Villani, Edward Carpenter, George Moore (2006) suggested the development of an interactive, photorealistic 3D computersimulated laboratory in microcontroller technology for undergraduate instruction. The virtual lab was implemented using interactive 3D computer animation technology commonly found in computer video games. Maya 6.5 (Alias TM) software was used to model the 3D lab components and to animate their functionality and OpenGL, C++ programming language ${ }^{[10]}$.

B. Aleksandrija Aleksandrova, Nadezhda Nancheva (2007) suggested the use of experiments based on computer simulations. They used movies and video clips as an instrument for visualization the act of magnetic field on moving charge. students had the ability to make connections between real-life phenomena, concrete and the abstract ideas and models of physics. They can create visual representations of their model and display it directly on the video scene. They also can combine images from different video frames and modify the presentation of motion on the screen. The excellent visualization effect of special interest with the description of electromagnetic phenomena represents the main benefit of computer simulations. Because of the effects of magnetic field on moving charge and Hall effect through action of abstract field that is hard to imagine.The researchers used Web based technology. Web pages were created by Microsoft FrontPage, ArcSoft Photostudio 5.5 and Microsoft Photo Editor. The presented laboratory exercises can be used in addition of real laboratory experiments. It can also be profitably used by the distant learners. It is important to accept in mind that the actual reality is inevitably more complicated than the virtual one. In spite of the simulation exercises are very good as an educational addition, they could not recompense the classical education ${ }^{[11]}$.

C. A.A. Eman, F.K. Naglaa(2010) suggested an educational program for children under 6 years old, comparing both animated means, aiming to illuminate their effect on a number of cognitive visual and dynamic visualization skills and learning some rhythmic gymnastics skills via 3D animated movies. The programmer used 3D Max for designing a 3 dimensional animated cartoon through the following steps: Create Model, Install a Himation Biped, Lighting adjustment, Create Surrounding Environment ${ }^{[12]}$.

D. Emanuel Dahlberg (2011) suggested electricity in a 2D mechanics simulator for education. Thesis covers the process of simulating electricity in real-time together with a mechanics simulator, called Algodoo. The thesis also shows that it is possible to simulate electricity in real-time, and that physically correct conducting objects requires a lot of processing power, but can be simplified without losing too much correctness ${ }^{[13]}$.

\section{THE PROPOSED SYSTEM DESIGN AND IMPLEMENTATION}

The main goal of this paper is to describe the form design and explaining the implementation of the of 3D Simulation System for Physical Lab using Object oriented software 
engineering. The figure (1) illustrates that there are four phases. The first phase is analysis the 3DS which consists of several stages.
The second phase represents the 3DS design which also consists of several steps.

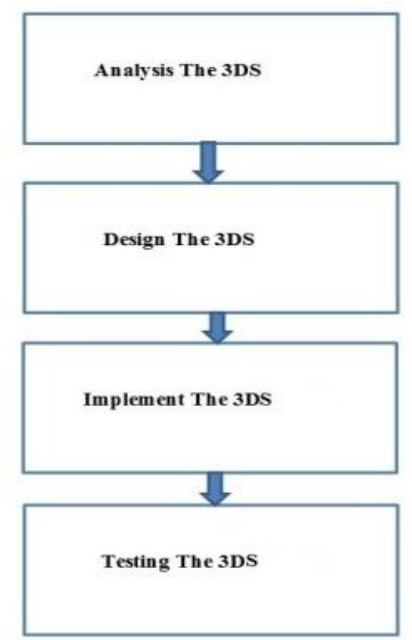

Fig (1) :General Overview Work steps using OOSE

The third phase is the 3DS implementation and the forth phase is the 3DS testing and evaluate it according to
OOSE. Figure (2) represents general overview to the programed design.

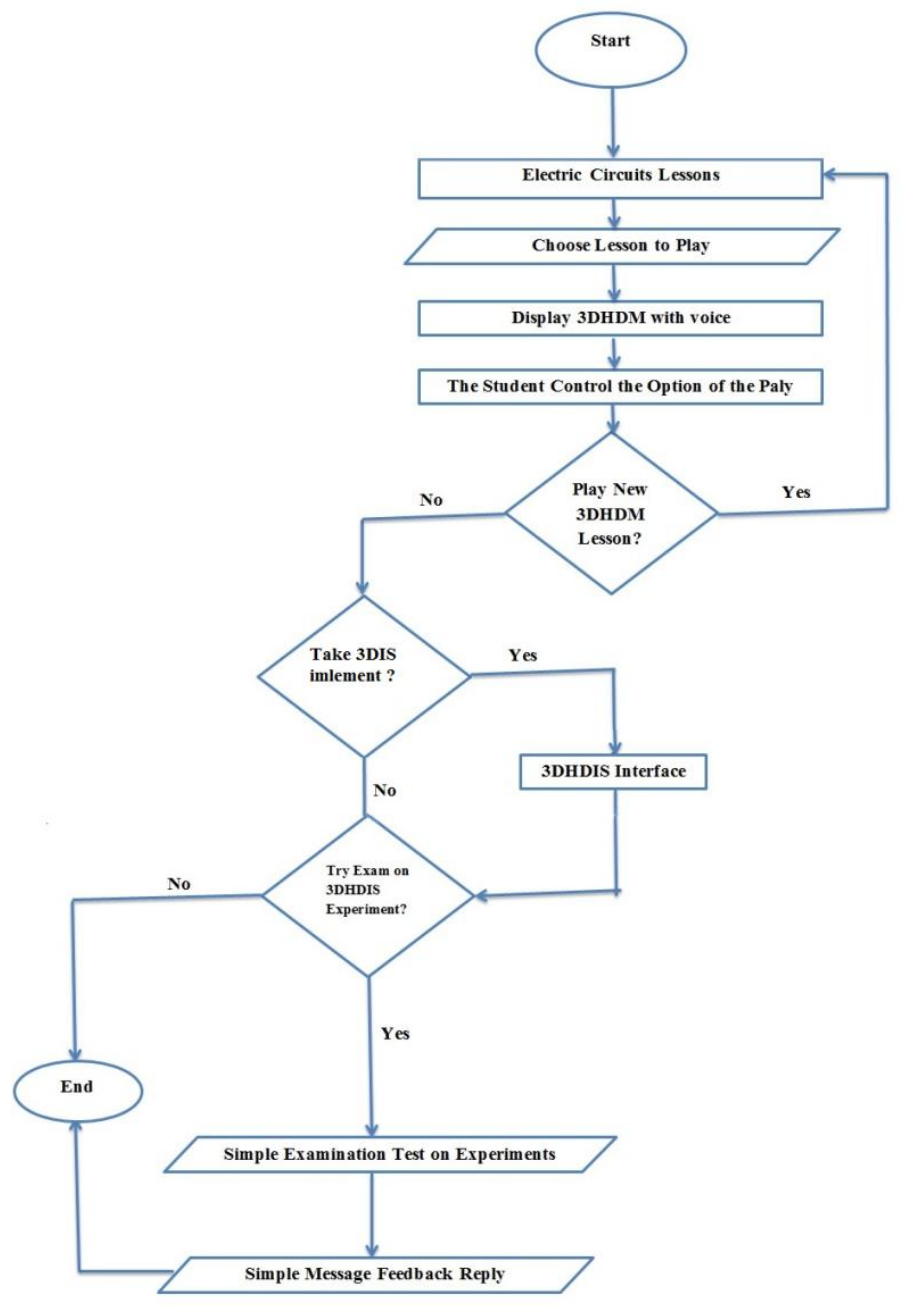

Fig2: System Design General Overview 


\section{THE DISCUSSION}

This part presents and discusses the results to evaluate the performance of the proposed system .

The proposed system is implemented using Autodesk Maya2014 ,MEL scripts Adobe Premiere Pro CC in additional of use of Tadween and Microsoft Visual Studio 2010

\subsection{D Modules Simulation System}

The 3DS starts with this user interface as shown in Figure(3) then after double click first interface will be run

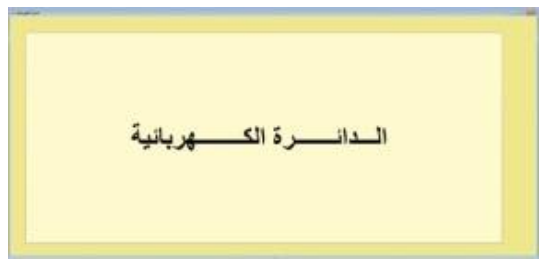

Figure (3) The started UI

\subsection{D Movies interface}

The 3DMs is illustrated in figure (4) The Window consisted of number of lessons in more detail which showing the view of the lesson .The Window media player of the program is playing the electric circuit movie. While figure (5) represents the message of the second stage.

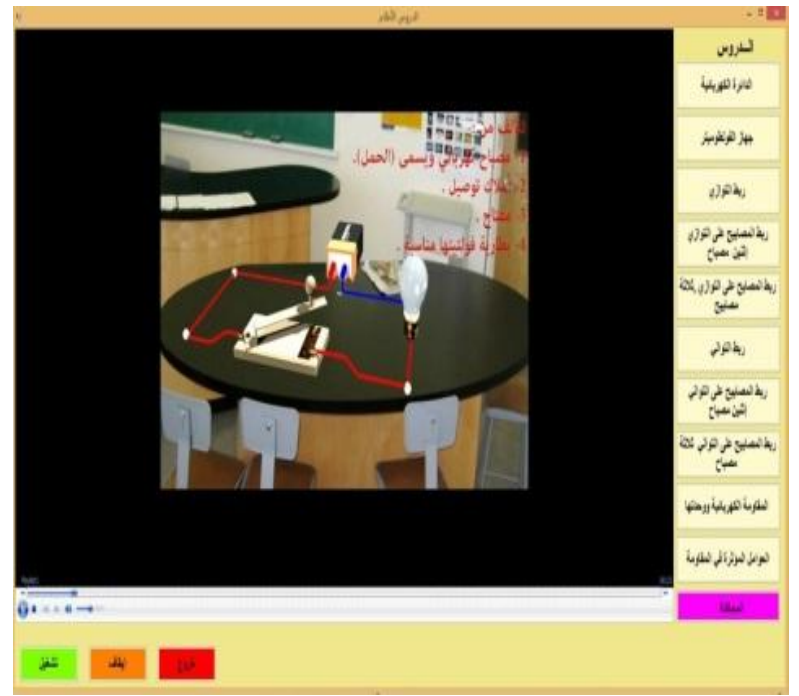

Figure(4) The 3D Movies Interface with displaying movie

After stopping the movie there is a message will be appeared for starting the instructional simulation for the selected subject as seen in figure (5)

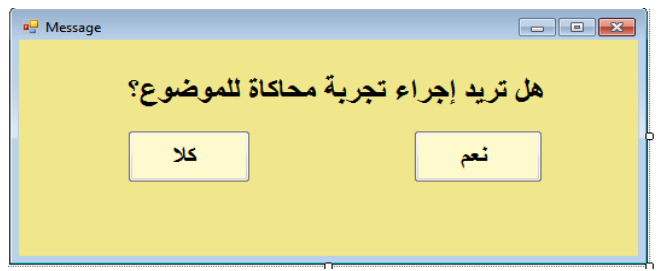

Figure (5) Message

\subsection{The 3D Simulation}

The figure (6) represents the simulation of electric circuit(EC). The plug was represented in two simple different manners to show the close and the open of the electric circuit
.The simulation was represented without voice recording not like the movie interface which contain sounds has been recorded in the each movie.

The mathematical part of the 3DS will be in the display window, The student makes his own selection .Choose either electricity, resistance or voltage and then compute .Flashing label has been used beside every item to let the student notice where he can put these values. The animation has been used to explain the movement of electricity in wire and the effect of electricity on the needle of the voltmeter.

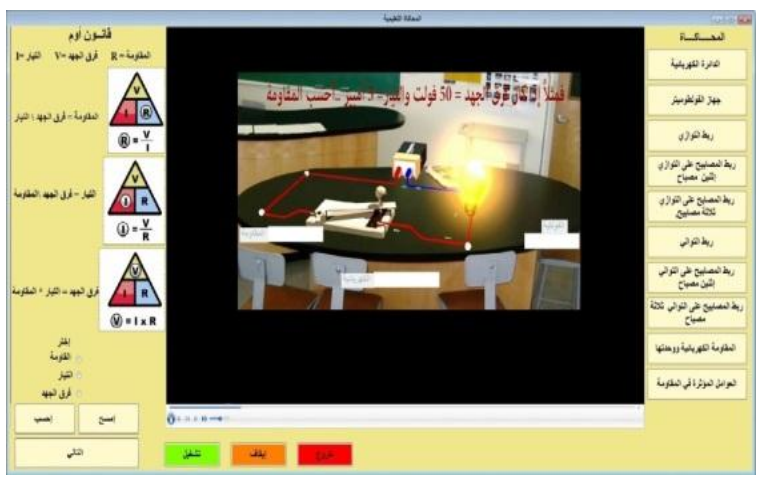

Figure (6) 3DM Simulation of Electric Circuit

The rules that have been used in the 3DS are added in an attractive colors based on 3D User Interfaces theory ${ }^{[14]}$ to let the student observes the different of each rule state. The student has the ability to rewrite on instructional interface numbers and chooses to test or see the results that he has got by using the pencil and a notebook.

\section{The results based on Ohm's Rule}

Tests are clearly indicated by using randomly numbers as show below in Table (1), Table(2) and Table (3 for electric circuit and Ohm's law only.

Table (1) Compute Resistance $(\mathbf{R})$ Resistance $(\mathbf{R})=$ Potential difference (V)/ Current (I)

\begin{tabular}{|c|c|c|}
\hline Resistance & Potential difference & Current \\
\hline 6 & 3 & 0.5 \\
\hline 12 & 6 & 0.5 \\
\hline
\end{tabular}

Table (2) Compute Potential difference (V)Potential difference $(\mathbf{V})=$ Current $(\mathbf{I}) *$ Resistance $(\mathbf{R})$

\begin{tabular}{|c|c|c|}
\hline Potential difference & Resistance & Current \\
\hline 12 & 6 & 2 \\
\hline 96 & 12 & 8 \\
\hline 45 & 90 & 0.5 \\
\hline
\end{tabular}

Table (3) Compute Current (I)Current (I) = Potential difference $(\mathrm{V}) /$ Resistance $(\mathbf{R})$

\begin{tabular}{|c|c|c|}
\hline Current & Potential difference & Resistance \\
\hline 0.25 & 220 & 880 \\
\hline 0.4 & 36 & 90 \\
\hline 0.2 & 36 & 180 \\
\hline 0.5 & 3 & 6 \\
\hline
\end{tabular}

\subsection{Test Simple Examination Interface}

Testing Phase will be simple twenty questions as multi choices quiz through choosing the right answer. The program uses Arabic language since the program is suitable for third $3^{\text {rd }}$ class at Iraqi School. The figure( 7) represent the first question of testing phase. If the answer was either right or 
wrong, a different text message will be appeared in each situation to let the student know if he answered correctly or not .For an example if the student choose the wrong answer for the figure (7) as shown in figure (8) .The message shown in figure (9) will be appeared.
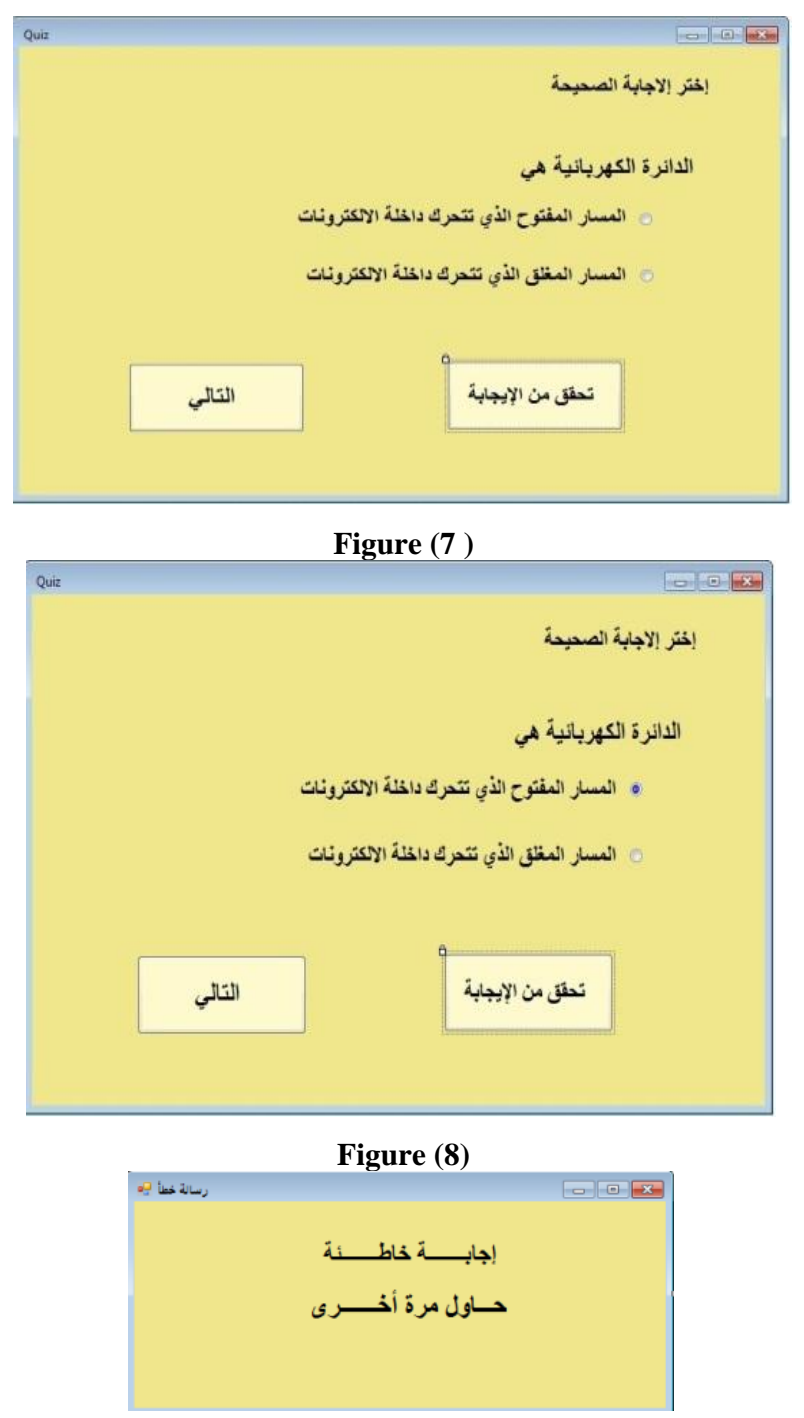

Figure (9)

If the student's answer was correct.The message in figure (10) will be appeared. These alert's message will be appeared after each answer.

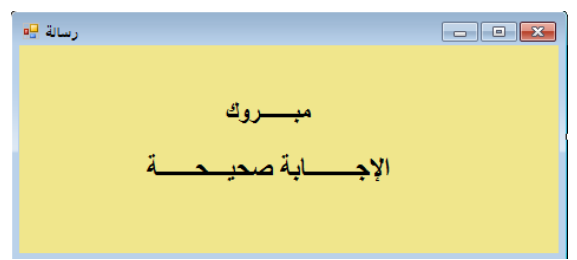

Figure (10) represent correct message alert

\section{THE EVALUATION}

The evaluation depends on the system's adopting way for the needs of the research work through evaluating the achieving the functionality and the usability of the adopted system based on OOSE. The 3DS system is very easy to use without training .It introduced simulation for physics experiments . The simulation are successful in implementing the experiments. Performance criteria is the response time is fast (Short time for executions).

There is no need for deploying cost since it is easy to use , Upgrade cost is good because it depends on high computer's Specifications ,there is no need for maintained cost and developing cost is relatively. It depends on the amount and the type of developing.Maintenance criteria depends on how The 3DS is easy to extensible, high readability, low modifiability, tractability of requirement is easy. End User Criteria represents the 3DS's utility is high and supports students study ,high usability.

\section{CONCLUSIONS}

The paper presents the 3DS System for 3D Animated for Physical Lab using Object Oriented Approach. It uses different digital multimedia techniques of computer and programs in addition to the use of OOSE in design and implementation. The student has the ability to enter his values and make repeatable computations . Ray tracing algorithm is used in a successful manner to introduce realistic images and pipeline graphics model are used in Autodesk Maya 2014 successfully. Using Tadween to accept the Arabic writing with adobe premiere pro $\mathrm{CC}$.

\section{SUGGESTIONS AND RECOMMENDATIONS}

- It will be recommended to develop the system to expand to have wide scope of science.

- The system could be online as a suggested work for the future.

- In future work the proposed system could be as mobile application.

\section{REFERENCES}

[1] M. Evison, Educating the next generation., vol. 48, no. 4. 2008, p. 196; author reply 196.

[2] K. L. Mcclarty, P. M. Frey, and R. P. Dolan, "A Literature Review of Gaming in Education Research Report,” Pearson, no. June, 2012.

[3] I. Halloun, Schematic modeling for meaningful understanding of physics, J. Res. Sci. Teach. 33, 1019 (1996).

[4] Lim, C.P.,(2007).Effective integration of ICT in Singapore school : Pedagogical and policy implications .Educational Technology Research and Development ,55(1),83-116

[5] Gibbons, A. S., Richards, R. R., Hadley, J., and Nelson, J. (2003). "The design of model centered instruction: a manual for the design of instructional simulations." In: Center for Human-Systems Simulation Technical Report, National Engineering and Environmental Laboratory. Idaho Falls, ID, Idaho.

[6] https://jcallisterdesign.wordpress.com/year-1/unit-66-3dmodelling/assignment-one/task-one/introduction-to-3dmodelling/explaining-geometric-theory/

[7] http://www.3dtotal.com/tutorial/1754-maya-modelingpolygonal-modeling-theory-by-jahirul-amin-characterface\#.VgqQiflVhBd

[8] Young-Suk Shin, (2002), Virtual Reality Simulations in Web-Based Science Education. 
[9] Maureen C. Stone,StoneSoup Consulting, SIGGRAPH 2001, (August 12, 2001), A Survey of Color for Computer Graphics, ( Course 4)

[10] Pavlos Kallonis, Demetrios G Sampson (2010), Implementation of 3D Virtual Classroom/Simulation for Teacherscontinuing professional development .

[11] Emanuel Dahlberg , (2011) , Electricity in a 2D mechanics simulator for education.
[12] Kamsin, A. (2007). Integrated 3D Multimedia Web Based Application in Biology: A Prototype. Computer Graphics, Imaging and Visualisation (CGIV 2007), 126132.

[13] OutStart, Inc.,2006, Best Practices for Creating ELearning100 Colonial Center Parkway Suite 400 Lake Mary, Florida 32746 407.548.0444 www.outstart.com

[14] Doug A.. Bowman , Ernst Kruijff , Josseph J LaViolla , Jr. , Ivan Poupyrev , (2005),3D User Interfaces Theory 